\title{
Book Review: "Biometric and Intelligent Decision Making Support"
}

\author{
F.G. Filip
}

\author{
Florin Gheorghe Filip \\ Romanian Academy, \\ Calea Victoriei 125, Sector 1, \\ Bucharest, Romania \\ ffilip@acad.ro
}

\section{Biometric and Intelligent Decision Making Support}

By Artüras Kaklauskas

\author{
Intelligent Systems Reference Library (Janusz Kacprzyk, Lakhmi C. Jain, Series Editors) \\ Springer, ISBN 978-3-319-13659-2, 236 pages \\ http://www.springer.com/us/book/9783319136585
}

This book is meant to familiarize the reader with the state-of-art of research and application results obtained in the domain of Intelligent Decision Support Systems (IDSS), biometric technologies and their integration. The author of the book is prof. A. Kaklauskas, the head of the Department of Construction Economics and Real Estate Management of the Vilnius Gediminas Technical University, Faculty of Engineering, Vilnius, Lithuania, a reputed author in the domain of Intelligent Decision Support Systems, Multi-criteria Decision Making (MCDM) methods and their applications in the construction, property management and the related fields. In the book, the author makes an exhaustive review of results reported in the domain literature and presents a detailed account of his practical achievements as well as his colleagues'.

The book is composed of seven chapters as follows.

Chapter 1, entitled "Introduction to Intelligent Decision Support Systems", sets the stage for the following chapters. It introduces the main concepts of the domain and pays a special attention to Artificial Intelligence (IA)- based advanced methods and their integration with Data Base Management Systems (DBMS) and Human- Computer Interface (HCI).

Chapter 2, entitled "Intelligent Decision Support Systems", reviews basic concepts of several new subclasses of IDSS, such as recommender, advisory and expert systems, data and text mining. The integration of Artificial Neural Networks is addressed too. A big part of the chapter provides abundant information about specific aspects of biometrics-based DSS, such as voice and speech recognition, expression analysis and so on. Two very modern concepts and associated technologies, namely Ambient Intelligence and Internet of Things, are described in details. Several other subclasses of solutions based on various technologies, such as Genetic algorithms, Fuzzy and Rough sets, Computer vision, robotic systems and so on, are surveyed in the final part of the text of the chapter.

The remaining part of the text contains the presentation of various conceptual and application results obtained by professor Kaklauskas and his colleagues. Chapter 3 introduces the model of passive house to be used in a qualitative analysis and design of the corresponding intelligent system.

Chapter 4 contains the results obtained in biometric and self-assessment of the student progress system and describes two relevant case studies. 
Chapter 5 addresses the subject of analysis of user's emotions and work productivity by using the state of blood circulatory system and describes a web- based biometric computer mouse advisory system.

The content of chapter 4 is complemented by the interesting solutions presented in chapter 6 , which addresses student progress assessment by using an intelligent pupil analysis and presents two case studies.

Chapter 7 addresses also the education domain and presents recommender-system-based solutions meant to increase student productivity.

One book addresses two modern research domains: intelligent and integrated decision support systems and biometrics-based human-computer interface. It can be explained, in my view, by the huge effort made by the author and his colleagues to find solutions to problems of the real world. The presentation of the research and application results obtained by the author and his colleagues over the years is a special merit of the book, beside the variety of the topics addressed. Consequently, I warmly recommend the book to PhD and MSc students and instructors, as an up-to-date source of knowledge. I think the book can also be a valuable tool for those consultants who are willing to master new concepts and technologies in order to get a competitive advantage on the market of computer applications.

\section{F. G. Filip}

Romanian Academy

Bucharest, Romania 